\title{
La interacción Universidad - Sociedad. Un nuevo concepto en la Educación Superior
}

\author{
The University Society interaction. A new concept in Higher Education
}

Recibido febrero 2021 Arbitrado marzo 2021 Aceptado abril 2021

Publicado mayo 2021

\section{Miguel Ángel Heredia Chumacero}

miguelheredia@gmail.com

Código ORCID: 0000-0003-4853-3810

Universidad Autónoma “Tomás Frías”, Potosí, Bolivia

\section{Resumen}

El artículo hace parte del trabajo de investigación denominado "Modelo Educativo basado en la interacción social universitaria para contribuir a la interacción universidad sociedad desde la materia de Proyectos en la Carrera de Economía de la U.A.T.F.", y tiene como propósito revelar la importancia de la interacción de la universidad con la sociedad, para formar profesionales según las exigencias y necesidades de la población, desde el enfoque histórico cultural. La estrategia metodológica del artículo, se apoyó en el primer capítulo de la tesis doctoral citada, buscando, por tanto, el desarrollo de tareas asociadas a la temática objeto de análisis, tales como: 1. Valorar la fundamentación epistemológica sobre la relación UniversidadSociedad; 2. Valorar la formación profesional en el marco de la relación Universidad-Sociedad; y, 3. Caracterizar la formación profesional. Entre los métodos teóricos utilizados, se tienen: el método históricológico, el método de análisis y síntesis; la modelación, el enfoque sistémico-estructural-funcional, y el ascenso de lo abstracto a lo concreto. El alcance estará dado mediante la interacción universidad sociedad en la Carrera de Economía.

\footnotetext{
Abstract

The article is part of the research entitled " educational model based on social interaction university to contribute to the interaction between universities and society from the Project area at the Faculty of Economics of the UATF " and has purpose is the importance of interaction between the university and society, to train professionals according to the demands and needs of the population, from the cultural historical approach, the methodological strategy of the article, was based on the first chapter of the thesis above, looking therefore development tasks associated with the subject under analysis, such as: 1 . Assess the epistemological foundation of the relationship between University and Society, 2. Rate vocational training as part of the University- Society, and 3. Characterize training; Among the theoretical methods used, we have : the historical and logical method, the method of analysis and synthesis, the modeling, the systemic structural-functional, and the ascent from the abstract to the concrete.
}

Palabras clave:

Interacción; universidad; sociedad; educación superior; formación profesional

\author{
Keywords: \\ Interaction; \\ university; society; \\ higher education; \\ vocational training
}




\section{INTRODUCCIÓN}

L

a actividad económica y social en el quehacer humano requiere necesariamente de una ciencia económica que responda a las verdaderas necesidades y problemas de Bolivia. El economista en ese contexto tiene un compromiso humano - conciencial, que debe responder al desarrollo humano sostenible, en términos amplios, porque cada especialidad buscará en su accionar profesional respuestas a las necesidades de la población, acorde al desarrollo técnico científico que se encuentra el mundo globalizado.

Los procesos docentes educativos que enfrentan las universidades bolivianas por las condiciones económicas, sociales y técnicas esencialmente, son aislados de sus entornos naturales, con los que casi no interactúan. Esta es una verdad donde todos nosotros somos responsables de tal aislamiento, porque pretenden ser un mundo de individualidades, y consideran que así como están sobreviviendo a todos los eventos.

La presente investigación busca que la universidad que forma economistas, los mismos encuentren en la INTERACCIÓN UNIVERSIDAD-SOCIEDAD los mecanismos adecuados para mejorar los procesos docentes educativos, quienes están compuestos por tres componentes esenciales, pero que por lo observado, estos están siendo descuidados en su relación dialéctica.

Estos componentes son: El académico, el investigativo y la práctica laboral investigativa, el último permite que el proceso docente educativo se relacione con la sociedad, como un mecanismo de retroalimentación y además de convertirse en el medio por el que la sociedad recibe los resultados de la investigación para mejorar sus condiciones de desarrollo.

La relación dialéctica y productiva que se debe establecer entre el estudiante y el docente, permitirá una formación académica en concordancia con los objetivos de la carrera, estableciendo un conocimiento con habilidades; donde, la investigación, que es una tarea del docente, como del estudiante; o ambos conjuntamente, debe buscar esencialmente una actividad productiva en el proceso docente educativo, de manera que la formación alcance niveles creativos enfocados a nuevos 
problemas, que enfrentara el estudiante, como el docente, en la relación que se deberá establecer en la práctica laboral investigativa, desde el punto de vista de Interacción UniversidadSociedad.

\section{Antecedentes y Gestión de la Interacción Social}

En Bolivia, existe el Reglamento General de Universidades y en su Artículo 19 dispone que toda universidad debe tener su Reglamento de Interacción Social con el objeto de normar la organización, desarrollo y funcionamiento de las actividades de Interacción Social. En su Artículo 62 enfatiza puntualmente que las universidades en concordancia con el modelo académico vigente, deberán contar con instancias responsables de promover la interacción social y la difusión cultural. Además de ser entidad responsable de diseñar, desarrollar, evaluar y difundir los resultados de programas, proyectos y actividades que mejoren en forma progresiva y continua la relación de la universidad con la sociedad.

La Interacción Social se desarrolla en el marco de la Responsabilidad Social Universitaria, con un nuevo enfoque en el momento de interactuar con la sociedad. Una manera distinta de reconstrucción de las relaciones sociales y subyacentes en principios como la responsabilidad social, actitud de servicio, honestidad, solidaridad, promoción de la salud y la defensa del medio ambiente. En consecuencia, la extensión o interacción social, equivale al Servicio Ecológico.

En concordancia con la filosofía, misión y modelo educativo de la Universidad, el Servicio Ecológico se concibe

como la acción concreta que cada estudiante deberá desarrollar en la comunidad, por medio de actividades y proyectos que permitan integrar de manera estructural la formación técnica, conocimientos y visión ecológica adquirida en su formación académica, en beneficio de la sociedad. (s.n)

En esta interacción, el estudiante logra contrastar su conocimiento teórico en un contexto real y por ende, desarrollar habilidades y destrezas para ser un profesional competente. 


\section{Interacción Universidad - Sociedad}

El apoyo a los Estudios Superiores debe ser una tarea y preocupación permanente de los poderes públicos. Un pueblo sin Universidad ya no es posible concebir en estos días.

El concepto de Universidad se pierde en el tiempo y espacio, en las corrientes evolutivas de la Edad Media y en la época moderna hasta nuestros días, donde la Universidad se manifiesta como un foro luminoso que irradia ciencia y cultura para el bienestar de la humanidad.

La Universidad es considerada como un organismo de Estudios Superiores en todos los países civilizados y su aporte al desarrollo de los pueblos es muy significativo y trascendental. El grado de desarrollo que tienen las ciencias en la actualidad se debe en gran medida al trabajo que realizan las universidades.

El presente artículo se propone abstraer la necesidad de los últimos años y acontecimientos que pasaron en la casa de estudios superior, y ver que la Universidad no se brindó a su misión y visión que fue creada, la de tener una relación Sociedad - Universidad, por diferentes aspectos.

Como se vio en los últimos movimientos sociales, y la misma necesidad de la sociedad de resolver diferentes aspectos, en diferente índole; la Universidad siendo una Institución, casa matriz que alberga distintos ámbitos sociales, tecnológico, científicos y de investigación, por el momento no hizo nada más que dedicarse a tratar de resolver sus propios problemas internos, y solo se quedó como un ente estático, cabe recalcar que teniendo diferentes unidades académicas y cada una de ellas debería de tener los diferentes centros de investigación, científico, tecnológico y sacar diferentes respuestas y soluciones a los problemas que día a día sufre la sociedad.

Por otro lado los diferentes profesionales que trabajan y se forman en la universidad no tienen una fluida interacción, solo se dedican a sacar folletos y libros que no resuelven en nada la problemática; como una verbigracia pueden ver los universitarios de las diferentes unidades académicas que solo hacen sus prácticas en la misma universidad y los que tienen relación con diferentes instituciones no se les toman en cuenta como elementos de la institución, más un solo lo sutilizan como mensajeros u/o 
secretarias; también los conocimientos aprendidos, no son aplicados en la realidad.

Tendrán que tomar en cuenta algunas premisas para poder entender mejor el problema del presente estudio:

- La Misión Social de la Universidad se ha ido perdiendo.

- La función de extensión como expresión académica debe revalorar sus aportaciones cualitativas al proceso educativo. (No asistencial)

- Las funciones de docencia, investigación y extensión en las universidades operan de manera poco integrada.

- El Servicio Social se ha desvirtuado. Los programas de extensión y difusión:

- No responden a un programa bien estructurado.

- Dejaron muchas veces un saldo de frustración en las comunidades.

- Son más iniciativas individuales que proyectos institucionales.

- Guardaron muy poca o ninguna relación con las tareas docentes e investigativas y con los planes y programas de estudio. (Extracurriculares).

\section{La Educación}

- La universidad es parte del sistema social, creado por la misma sociedad, debe influir sobre ella y propiciar su cambio.

- La extensión universitaria es realmente una comunicación del quehacer universitario en diálogo permanente con la sociedad.

- Implica un proceso de interacción y reciprocidad en la relación Sociedad - Universidad.

Pero la misión y visión de la Universidad no solo se debe basar en dar un título y grado sino en ver la realidad y los problemas que existen en la sociedad y tratar de resolverlos.

La Universidad evolucionó a lo largo de la historia, su tendencia a la formación de una unidad espiritual y de asimilación de las ciencias, en unión con profesores y estudiantes donde no existen fronteras ni límites de nacionalidades, ni diferencias político - ideológicas. 


\section{Caracterización Histórica de la Interacción Social}

\section{Marco contextual del entorno universitario}

La formación de profesionales trae como consecuencia un mejor desarrollo económico, pues permite establecer niveles óptimos de servicios y producción, que es el sustento de vida de cualquier país. Pero la enseñanza técnica que logre habilidades, conocimientos, destrezas y valores tiene un precio alto, esencialmente por el costo de los medios de enseñanza, expresado en los equipos e insumos que se requieren en este proceso. Superar éste problema tiene sus dificultades que deben enfrentar creativamente. Hasta ahora se utilizan los laboratorios que van quedando obsoletos por el avance de la ciencia, y por consecuencia lógica, la formación profesional responde a esas innovaciones y desarrollo tecnológico actual.

Coadyuvante con ese escenario, el modelo económico predominante que se vive, no encuentra las ansiadas soluciones a los problemas, más bien los agudiza, trayendo como consecuencia una mayor pobreza y sistemas educativos desprendidos de su realidad social, con una recesión que no permite una actualización tecnológica que active una verdadera interacción social. Las intenciones en estos temas de parte de la universidad son claras. Sólo con estudiar los últimos congresos universitarios, como el VI llevado a cabo en la ciudad de Tarija en 1983, posterior a la apertura democrática, busca afanosamente reorientar su papel, "unir la Universidad con el pueblo trabajador" (Comité Ejecutivo de la Universidad Boliviana, 1985, s.n.).

De la fuente mencionada anteriormente conviene destacar lo siguiente: "La Universidad deberá situarse en el conocimientoacción de los problemas concretos de la sociedad". (s.n.).

Es quizás aquí donde se pone en claro la necesidad de permitir prácticas laborales enlazadas con la interacción social, resoluciones que salen de los congresos universitarios, pero muy pocas unidades académicas ponen en práctica estas propuestas, por todas las condiciones anotadas anteriormente.

La Organización de las Naciones Unidas caracteriza la actividad 0 fin de cualquier entidad organizada como: Producción = Operación + Mantenimiento. (Tavares, 1994). 
Los profesionales deben dominar las tareas de mantenimiento y operación, dentro de un espíritu de perfil amplio y currículum flexible, la formación de habilidades y destrezas en las dos actividades, teniendo como fines esenciales lo siguiente:

- Garantizar diseños que sean eficientes, seguros y rentables con el tiempo.

- La reducción de la paralización de equipos que afecten las operaciones.

- Garantía de funcionamiento de las instalaciones de forma que los productos y servicios atiendan a criterios establecidos por el control de calidad y estándares preestablecidos.

Estos aspectos que son de carácter puramente técnico, son consecuencia de un conocimiento adquirido en la práctica diaria de la empresa, y tienen una dinámica al igual que la Revolución Técnico-Científica; no son fruto de razonamientos sesudos que nacen de un laboratorio, nacen, más bien "sobre la relación entre el conocimiento y la práctica, entre el saber y el hacer"(Mao, 1977) y aún más, esa experiencia hoy se liga a la necesidad de establecer normas que cuiden y preserven el medio ambiente, pero como ya se advirtió, la ejecución de estas tareas requiere de conocimientos amplios, que sólo será efectivo en la medida que se encuentre alternativas que incentiven la investigación, además, no olvidemos que Bolivia es un país que tiene situada gran parte de su actividad económica en zonas de gran altura.

Este contexto característico de un país pobre, con un desarrollo económico, político y social por debajo de los estándares óptimos, obliga a encontrar alternativas que encaren soluciones dentro de nuestras limitaciones.

En resumen podemos afirmar que el profesional es un engranaje importante del desarrollo económico, siempre y cuando se permita en su formación alcanzar las competencias profesionales que respondan a las necesidades sociales del entorno; cumpliendo los mandatos del Estatuto Orgánico de la Universidad Boliviana que es interactuar adecuadamente, en una búsqueda de ofertar soluciones a los problemas concretos en beneficio de la sociedad. 


\section{Fundamentación Teórica de la Interacción Social}

\section{Universidad Popular. Una Forma de Interacción Social}

La Universidad Popular nace como una instancia de comunicación con el pueblo trabajador, es el primer intento de realizar la interacción destinada a resolver las problemáticas del país, pero esta no se concretiza con el tiempo en una verdadera relación pueblo-universidad.

Pese a los últimos cambios económicos que se vive en el mundo, el ser humano no resuelve completamente sus relaciones sociales y económicas, permitiéndose una sociedad estable, basada en la equidad, la justicia, la independencia, la libertad; principios que garanticen la perpetuidad de la raza humana. En esa constante lucha por la vida, nace como una dádiva del sector estudiantil en el año 1928 un Estatuto Orgánico para "Hacer efectiva la alfabetización y la extensión universitaria mediante Ligas de alfabetización Y La Organización De Universidades Populares, gratuitamente atendidas por universitarios" (Medina, 1999). De esta manera se aprobó en Sucre el 22 de enero de 1930 el Estatuto De Creación De Las Universidades Populares De La Federación Universitaria Boliviana.

La Universidad Popular se crea como un servicio de extensión al sector obrero y tiene un funcionamiento irregular; bajo la dirección del sector estudiantil, con políticas características de la época, como era la formación de dirigentes sindicales obreros esclarecidos y respaldar a las universidades en la conquista de su autonomía, avanzando en la conquista de otras metas; como es la defensa de las fronteras, socialización progresiva de la propiedad privada, nacionalización de las minas, el petróleo y lucha contra el latifundio y dotación de tierras a los indios (Medina,1999).

Las realizaciones de la Primera como Segunda Convención de la Federación Universitaria Boliviana fueron saltos cualitativos fundamentales; no sólo en la marcha del movimiento universitario del país, sino también para el movimiento laboral boliviano organizado. Son estas las instancias las que adoptan para sí el papel de "custodios" de los intereses populares y, particularmente, de los obreros (Medina, 1999).

La revolución de abril de 1952 con la ascensión al poder del Movimiento Nacionalista Revolucionario, conllevó a una serie de 
cambios, esencialmente ejercidos por la presión del pueblo boliviano. La Universidad Popular, por ser una dependencia de la Universidad; se regiría con su Estatuto y disposiciones, y el Reglamento aprobado, impartió el estudio de las ciencias sociales, económicas, políticas y jurídicas a los trabajadores manuales de las industrias, además de la enseñanza Técnica que les permita contribuir al progreso de Bolivia, fomento entre sus miembros la cultura general, manifestaciones científicas, artísticas, literarias y deportivas. Contempla igualmente otro acápite de interés de los campesinos al señalar la divulgación de nociones básicas de la Agropecuaria, incluyo las relativas a la prevención y curación de enfermedades vegetales y animales. La Universidad comprenderá a partir de ese momento las siguientes partes características:

- Escuela de capacitación Sindical, Política y Cultural.

- Escuela de Artesanía y Técnica Industrial.

Algunos resultados de estudios en Bolivia:

El Estatuto Orgánico de la Universidad Boliviana, que en su primer artículo reza: "La Universidad Boliviana es un sistema de educación superior constituida por las universidades públicas autónomas e iguales en jerarquía" (Comité Ejecutivo de la Universidad Boliviana, 1985); dentro de ese principio se establece en la Nueva Ley Fundamental de la Universidad Boliviana (1975), en el artículo 51 que los Politécnicos Universitarios son "cursos regulares de extensión" (s.n.), además, en el artículo 98 expresa: "La Extensión Universitaria es el conjunto de servicios que la Universidad ofrece a la colectividad para hacer asequibles a los diversos sectores de la población los beneficios de la cultura, de la investigación y la enseñanza, contribuyo al desarrollo de la comunidad. Debe ser programada por cada Universidad y dirigida en su acción al mejoramiento de la comunidad, por equipos interdisciplinarios" (Nueva Ley Fundamental de la Universidad Boliviana, 1975). Además, en el mismo capítulo artículo 99, enuncia que la "Extensión Universitaria depende del Vice rectorado" (s.n.). También paradójicamente se enuncia que "no estará vinculada a finalidades político-partidarias" (s.n.).

Se observa que la Extensión Universitaria, era el modelo de relación con su entorno de la Universidad, modelo precario que 
toma nuevos rumbos hoy en día. Como consecuencia puedo concluir:

a) Que en una etapa inicial la Extensión Universitaria, ligada a la Universidad Popular, se caracteriza por la reivindicación los intereses nacionales ligados al pueblo trabajador, sufriendo transformaciones de forma, hasta llegar a ser una unidad de capacitación de mano de obra.

b) Nace una segunda etapa regulada por La Nueva Ley Fundamental de la Universidad Boliviana, dirigida a frenar el avance popular de esos años (1971); que en la teoría cumplía con algunos requisitos de interacción con los sectores de la población, pero esta división dependiente del Vice-rectorado cumplía funciones culturales, como presentaciones artísticas de diversos tipos, ya sean musicales, de pintura, poesía y quizá en algunos casos eventos del tipo científico. Estas actividades no fueron sistematizadas, porque tampoco se estableció mecanismos que encaren un verdadero proceso de interacción social, peor aún, si este estaba centralizado en Vice-rectorado, como disponía la norma. Las unidades académicas conformadas por las carreras de formación profesional, no se veían involucradas, perdiendo ese contacto y deseo de dar sus servicios con esta división.

c) La tercera etapa, que actualmente se está viviendo, como consecuencia del VI Congreso de Universidades; el Estatuto Orgánico define la extensión como "la relación recíproca entre la Universidad y el pueblo" (Comité Ejecutivo de la Universidad Boliviana, 1985, s.n.) además, en el artículo 64 se expresa que "La Universidad Boliviana prestara asesoramiento en los campos tecnológico y científico cuando así lo requiera el gobierno Nacional y Local, el municipio y las corporaciones de desarrollo" (Comité Ejecutivo de la Universidad Boliviana, 1985, s.n.).

Es aquí donde se fortifica una nueva concepción de la extensión universitaria, que si interpreta mejor, cada unidad debería trabajar con programas planes y objetivos generales y específicos de la Universidad, en cada carrera y área de conocimiento, que no es una división centralizada, y debería ser un programa de cada unidad, para que así sus estudiantes puedan desarrollar la práctica laboral; esto queda claro en el documento de la secretaria académica "Bases epistemologías, teóricas, metodológicas y psicológicas del nuevo modelo académico", que 
en su parte correspondiente a: "Universidad-Sociedad y Estado"(Secretaria Académica del Comité Ejecutivo de la Universidad Boliviana, 1 983), en él se expresa: "En tal virtud es imperiosa la necesidad de que la universidad debe volcar su mirada" a sí misma, y a su entorno o contexto social. Volcar su mirada "a sí misma" implica la realización de una evaluación de la universidad, con respecto a todos sus elementos poniendo especial énfasis en las UNIDADES ACADÉMICAS (carreras), que permita establecer con objetividad la correspondencia entre las necesidades de la realidad social y la práctica académica de la institución (universidad), ello a su vez te permitirá puntualizar los "quiebres" entre las necesidades reales y el quehacer de la universidad que se operativiza a través, de las carreras, institutos, y centros de estudios de tercer nivel; estableciendo las causas que mantienen la distancia o "brecha" entre los Postulados, Principios, Fines y Objetivos de la universidad y su práctica real concreta: distanciamiento universidad sociedad"( Secretaria Académica del Comité Ejecutivo de la Universidad Boliviana 1983).

d) Dentro de ese marco de referencia, con bases claras para implementar un Nuevo Modelo Académico, la mayoría de las universidades no responden con su real implementación como una política de mejoramiento, esta situación deja a traslucir que los razonamientos, de ese VI congreso de universidades, tienen lineamientos y soluciones a lo que ahora denominamos la Práctica Laboral Investigativa, quedando todo esto como un simple enunciado teórico, además, se considera en el documento de la secretaria académica que hace "falta determinar las prioridades de las tareas que implica un análisis, una reflexión y una síntesis, y la posibilidad de formular alternativas de acción que incidan en ellas y la voluntad política de implementarlos, voluntad política qué deberá tomar en cuenta las condiciones objetivas y subjetivas; las que se expresan en la correlación de fuerzas de las clases sociales, al interior de la formación económico social actual; correlación de fuerzas que no deben ser analizadas 'in situ', también realizar un análisis prospectivo de las mismas"(Secretaria Académica del Comité Ejecutivo de la Universidad Boliviana, 1983). 


\section{Enfoque:}

Se afirmar que la excelencia es fruto de la interacción entre todos los componentes académicos, que respondan dialécticamente a la división natural del trabajo.

El modelo del presente trabajo busca en la relación Universidad-Sociedad, o planteada de manera particular, entre la carrera de economía con su entorno natural, encontrar métodos de enseñanza productivos o grupales, que busquen el desarrollo de las habilidades cognoscitivas de los alumnos, que suponen niveles superiores del pensamiento, consecuencia de una vivencia real en el entorno natural.

El docente no es más que un guía con una mayor experiencia, para enseñar y conducir el proceso y análisis, incorporado sus experiencias a problemas nuevos con carácter creativo, en cambio el estudiante construye sus conocimientos en las relaciones docente-estudiante, como vínculo de cooperación, en el quehacer de la interacción donde los problemas profesionales obligan a la investigación para dar respuestas adecuadas. El enfoque bajo esa circunstancia tiene un carácter de Didáctica Crítica.

\section{Tendencias}

La universidad como institución pública, y de carácter autónomo, permitió el desarrollo de actividades contestatarias a los modelos de estado, que nunca pudo ni puede proponer soluciones a las problemáticas políticas sociales y económicas del país. En ese acometido uno de sus propósitos primordiales es interactuar con el escenario de los profesionales que forma, para permitir una formación académica con habilidades y valores, que ayuden al desarrollo nacional con conciencia social. Este fin y objetivo no se pudo establecer, como derivación de políticas inadecuadas que partieron de un modelo centralizado.

Históricamente la Interacción Social Universitaria es un objetivo estratégico, que no está resuelto, pero que sí es de preocupación de todos los actores comprometidos con el cambio, impulsar su desarrollo e implementación, debe ser el propósito de todos y en especial como propuestas concreta del presente trabajo. 


\section{La universidad en una sociedad en trasformación}

Estos últimos años la sociedad boliviana ha reflejado su condición social conflictiva donde los patrones de dominación denominados coloniales o neocoloniales se han evidenciado nuevamente y no han sido aún superados, donde las diferencias culturales se encuentran entretejidas con las desigualdades sociales y económicas, razón por la cual las políticas interculturales implantadas desde fines de los años 80 no pudieron aplacar las prácticas discriminatorias.

Por otro lado, el discurso de la descolonización de la sociedad y de la educación ha bajado de tono del impulso que había tomado hace unos seis años atrás y, ante todo, en la práctica cotidiana, pocas nuevas vicisitudes y virtudes sociales y políticas se han revelado.

En todo caso, el nuevo escenario que vive la sociedad boliviana enmarcada bajo la nueva constitución política del estado y la nueva ley educativa Avelino Siñani - Elizardo Pérez (dejando de lado la inmensa cantidad de leyes para el efecto), pone la universidad ante diversos desafíos que a continuación se puntualiza:

Objeto y perspectivas de la educación superior y, en especial, de la universidad. La visión de universidad que orienta el proyecto actual parece conjugar la formación de recursos humanos con la recuperación de saberes y conocimientos expresados en las ciencias y las tecnologías, así como con la investigación que responda a las necesidades sociales de los pueblos (indígenas originario campesinos). Su objetivo es formar profesionales, desarrollar la investigación, democratizar el acceso al conocimiento, con un sentido crítico.

Estos objetivos se fragmentan al momento de considerar los procesos históricos diversos del país, con sus diferencias culturales exaltadas en los últimos años, lenguas, regiones geográficas, sistemas productivos y políticos tradicionales muy diversos, etcétera.

El campo de la educación superior. Hasta hace poco (los años 60 y 70) la universidad boliviana parecía cumplir su función transmisora de conocimientos, centrada en la enseñanza, con una población estudiantil relativamente reducida social y 
demográficamente (espacio casi propio de la clase media y alta, urbana, de la sociedad boliviana), en la que la investigación no ocupó un lugar central. Además, tenía un reconocimiento social, es decir una legitimidad, no sólo porque representaba el ascenso o aseguramiento social y cultural sino igualmente porque fue el espacio de las luchas sociales revolucionarias. Empero, desde los años 80 y sobre todo los 90 , el espectro se torna difuso con la ausencia de ideologías motoras de cambio, la institucionalización de una forma burocrática de funcionamiento, el ingreso masivo de la nueva población inmigrante rural-urbana, y la reiteración de un modelo universitario enseñante. Al respecto, algunos autores han hablado de crisis de la universidad (Santos, 2006; Rodríguez, 2000).

En todo caso, los marcos jurídicos descritos en este trabajo dejan evidente que el campo de la educación superior se diversifica. Está claro que desde ahora (siguiendo la ley) la universidad ya no es la única vía para formarse a nivel superior.

Primero, la formación de maestros (antiguas escuelas normales) que históricamente intentaba aproximarse a la universidad pública tiene su camino abierto hasta niveles universitarios ya que se proyecta claramente en una filiación vertical normalista, es decir incrementa en años de formación, de tres a cinco años, pero no rompe la tradición normalista y además mantiene la exclusividad de la función formadora de maestros, no dejando participar otras instituciones en la formación de maestros. No se sabe hasta qué punto esta política y práctica endogámica de la formación docente podrá mejorar la calidad de la formación de maestros a futuro.

Segundo, la formación técnica y tecnológica, paralelamente a la formación universitaria, se abre y amplifica en sentido horizontal de los sectores productivos y laborales, y permite asimismo la posibilidad de continuar hasta niveles superiores universitarios (ascenso vertical).

Tercero, la formación artística sigue el mismo sendero de la educación técnica.

Cuarto, se ratifica la formación universitaria pública y privada, en condiciones bastante diferentes.

Es decir que esta separación no es puramente administrativa sino tiene bases y consecuencias distintas mucho más 
significativas por la ruptura que reflejan ambos sub-sistemas en términos de concepción de la formación, los conocimientos, etcétera, hasta el no-reconocimiento de diplomas de un sistema al otro. Con la nueva ley, a estas universidades se adicionan las universidades indígenas y las universidades especiales de las Fuerzas Armadas y de la Policía, de las cuales se ignora sus cualidades.

La formación de maestros. La formación docente tiene una historia compleja y muy diversa, lo que llama la atención en la propuesta actual es el incremento en años de formación en paralelo a las universidades públicas y privadas, sin posibilidad de interactuar entre estas dos instituciones que pueden muy bien enriquecerse recíprocamente. Hay que recordar que en los años 1930 y 40 la formación docente de la normal de Sucre se integró a la Universidad San Francisco Xavier, sucedió lo propio con la normal Simón Bolívar de La Paz con relación a la Universidad de San Andrés, en un momento de su historia. Más recientemente, con la reforma educativa, algunos INS fueron delegados en su administración a las universidades públicas y privadas.

Lo que ilustra que la relación entre universidades y escuelas normales siempre oscilaron entre acercamientos y distancias, aun cuando estos movimientos fueron más de decisión política de los diversos gobiernos que del magisterio. A nivel internacional, las experiencias de formación docente son diversas que van desde sistemas endogámicos de formación como el caso boliviano (tradición normalista), pasando por sistemas mixtos hasta la integración completa de la formación docente en las universidades. En general, los docentes de secundaria se forman en las universidades y de niveles inferiores se forman en Institutos Superiores Pedagógicos, Escuelas Normales u otras reparticiones de las universidades con dos o tres años de formación.

Las formaciones técnicas y tecnológicas. El desarrollo de la educación técnica es un desafío muy grande e interesante en un país como Bolivia, sin un sistema productivo desarrollado y estructurante para esta formación. En este sentido hay que dejar en claro que, a nivel internacional, este tipo de formación está relacionado y condicionado históricamente por el desarrollo industrial y tecnológico de los países. Inicialmente escasas veces la formación técnica fue la generadora de sistemas de trabajo, más 
bien estos últimos fueron los inductores de la formación técnica. Un indicio de esto en Bolivia es la experiencia del Instituto Pedro Domingo Murillo de La Paz, muy reconocido y valorado pero no masivo. Así existen casos en Colombia, Brasil, Argentina, etcétera.

Por consiguiente la ampliación de la educación técnica en Bolivia está sujeta a cómo este país puede encarar su proceso de industrialización o al menos estructurar su sistema de trabajo o laboral que oriente la demanda de profesionales técnicos en los diferentes sectores (distribución horizontal) y niveles de competencias (distribución vertical). De otro modo, el camino por venir será costoso y no garantizará necesariamente su función laboral.

Ahora bien, esto puede variar de un sector a otro, por ejemplo, el sector terciario y de servicios, por la característica del país principalmente consumidora y consumista y no tanto productiva e industrial, la formación técnica de este sector puede tener (ya tiene) éxito (además no es costosa).

Los postgrados. El campo de la formación de los postgrados mantiene filiaciones paralelas en cada tipo de educación superior descrito. A nivel de las universidades públicas los Centros de postgrado, creados hace una década o más aproximadamente, deberán desarrollar más programas de formación que contribuyan a la especialización de recursos humanos y al desarrollo de la investigación como medio de producción del conocimiento científico. De acuerdo a las tendencias internacionales pronto se desarrollarán los postgrados de cada Facultad e incluso de cada Carrera, formando profesionales especializados en cada área profesional y de conocimiento y realizando investigaciones al nivel de maestrías y doctorados. Esta idea de especialización no debe confundirse con aquella que sirvió al nacimiento de las disciplinas académicas y científicas (siglos XVIII y XIX), pues actualmente la tendencia es más bien ir hacia la interdisciplinariedad y transdisciplinariedad (Gibbons, 1997). Lo que significa tener un trabajo cuidadoso en la formación base, en núcleos cognoscitivos y sus fronteras e interrelaciones con otras disciplinas o campos de saberes.

La investigación. A fin de avanzar en el desarrollo de la investigación, las universidades actuales del país deberán realizar un cambio importante en la visión de la propia institución. Hasta 
ahora, la universidad boliviana se caracterizó y se caracteriza todavía por su función de enseñanza y transmisora de conocimientos, el uso de manuales y otros libros que llegan de universidades extranjeras.

En parte, es esta función que explica el valor que toman los aspectos didácticos y pedagógicos en la educación superior, demostrados con el desarrollo de los programas de postgrados en educación superior: diplomados, maestrías e incluso existen doctorados en educación superior. Decía que el modelo universitario explica parcialmente el énfasis puesto en la enseñanza porque existen otros factores sociales y culturales tales como la masificación del estudiantado y además de origen más popular (en las universidades públicas) y las condiciones académicas con las que los estudiantes ingresan (Paz Soldán, 2011).

Entonces, el desarrollo de la investigación ¿qué lugar debe y puede ocupar en las universidades? La respuesta pasa por analizar, no sólo el modelo de universidad vigente (modelo napoleónico) y transitar hacia otra universidad que permita y facilite la producción de conocimientos (modelo universitario humboltiano), sino igualmente la ciencia y los conocimientos científicos que traen consigo las exigencias de la investigación en su naturaleza y sus caracteres.

Las políticas de conocimiento y curriculares. Este punto es muy poco abordado en la nueva ley ASEP, a diferencia de las pistas que se encuentran en la CPE y el Plan Nacional de Desarrollo de 2007. En realidad la ley parece reflejar una visión educativa restringida al componente institucional donde el curriculum se asimila a la estructura académica por niveles, que está muy claramente establecida. Sin embargo, el campo curricular es amplio y complejo que no puede dejarse en silencio, sobre todo para los niveles inferiores de la educación.

Es posible que las políticas culturales y de conocimiento en las universidades públicas y privadas sean difíciles de delimitar en el marco jurídico de la ley, pero, entonces la tarea del análisis y las decisiones en este dominio corresponden al campo de las propias universidades, las cuales "deberán" tomar en cuenta: 
- Los conocimientos y los saberes en todos sus estados (tipos de conocimientos), orígenes (de dónde provienen), propiedad (a quiénes corresponden), posiciones (que posición ocupan en las estructuras y jerarquías de enseñanza) y valores (cómo valoran los conocimientos las personas que enseñan y aprenden).

- Que la universidad es un lugar privilegiado en utilizar, distribuir y producir ciertos tipos de conocimientos y a través de ellos establecer ciertas relaciones sociales de dominación y también de "liberación".

- Que los conocimientos están relacionados a intereses e ideologías de grupos sociales, pero no deben reducidos a ellos.

- Que los conocimientos son contenidos formados en tiempos y espacios particulares que merecen ser tratados en su especificidad, mas no son únicamente eso, son también relaciones sociales.

- Que los conocimientos emergen de espacios locales y particulares donde los sujetos producen (por ejemplo las universidades o los pueblos indígenas) y que para ingresar a otros espacios necesitan reconocimiento y manejo apropiado, por eso, como decía Michel Serres, sus desplazamientos y trasferencias representan caminos inciertos (por ejemplo iqué conocimientos indígenas pueden ingresar a las universidades y en qué condiciones?).

- Que si bien se puede postular que el conocimiento es un bien público, la historia moderna y contemporánea del sistema capitalista y de mercado en que se vive, hace que los conocimientos sean tratados como bienes y objetos privados y comerciales.

Por último, ¿̇cómo analizar e intervenir en la relación entre conocimiento y sujeto? Todos saben que las personas son valoradas más o menos de acuerdo al tipo de conocimiento que manejan, lo cual tiene repercusiones en las relaciones de poder donde las valoraciones legitiman estas relaciones. Es decir: ¿Cómo desprender este vínculo: sujeto, saber y poder? 


\section{Universidad Autónoma Tomás Frías (U.A.T.F.)}

En forma oficial mediante Ley del 15 de octubre de 1892 nace nuestra Universidad Autónoma “Tomás Frías" (U.A.T.F.), sancionada por el entonces presidente Dr. D. Mariano Baptista. La actual Facultad de Ciencias Económicas, Financieras y Administrativas tiene su origen el Instituto Superior de Comercio que se fundó en Potosí el 22 de marzo de 1937; el 8 de octubre de 1937 se consolidó la autonomía de la universidad con la incorporación con la incorporación del Instituto Superior de Comercio que anteriormente dependía de la Prefectura Departamental; por resolución de IV congreso de facultades de economía del país, realizado en mayo de 1961, se adopta la denominación uniforme de FACULTAD DE ECONOMÍA; desde octubre de 1982 la universidades retoman el cauce democrático, oportunidad en la cual la facultad recobra su estructura actual con las Carreras de Auditoria, Economía, Administración de Empresas a nivel de Licenciatura y Contabilidad a nivel de Técnico Superior; por resolución N020-88 del Honorable Consejo Facultativo, se adopta la nueva denominación de Ciencias Económicas, Financieras y Administrativas, a partir del 15 de noviembre de 1988; La Carrera de Economía fue fundada el 21 de noviembre del 1946 (Centenario de la Universidad Autónoma Tomás Frías 1892 1992, 1993)

\section{Carrera de Economía}

La Política de Interacción Social de esta carrera ya está inmersa desde el Estatuto Orgánico de la Universidad Boliviana, la interacción social es la actividad que permite la relación recíproca entre la Carrera de Economía y su entorno socioeconómico; dicha actividad se desarrolla en estrecho vínculo con las actividades de investigación. Teniendo en cuenta que en el "Plan Curricular de la Carrera de Economía" (Universidad Autónomas Tomás Frías, 2002, pp. 38-39) cuenta con la práctica laboral, su finalidad es tener contacto con las instituciones tanto públicas como privadas para lograr una adecuada familiarización con los espacios laborares donde puedan desarrollarse profesionalmente los estudiantes, lamentablemente esto solo fue literal y nunca se llevó a aplicar, solo se quedó como documentación. 
De la misma manera tiene el sustento legal en la Ley 70 (Ley De La Educación Avelino Siñani - Elizardo Pérez, 2010) donde en su sección iv formación superior universitaria, el artículo 52 y 53 nos habla de la política de interacción social y promoción de las universidades.

Generalmente, las universidades latinoamericanas, y las universidades estatales de Bolivia tienen tres funciones definidas y establecidas, que la caracterizan y la diferencian; estas funciones son: la formación, la Investigación Científica y la Interacción social.

La universidad, (Boliviana, por la falta de inclusión y revalorización de los pueblos indígenas; además, de la demanda de un control social, discutible en cuanto a sus dimensiones de teorización y aplicación), no se ha preocupado por entrar en contacto con la sociedad mediante una interacción, limitándose a ser sólo emisor de información o reproductor de conocimiento. Reflejo de ésta crisis, son un claro ejemplo: las creaciones de universidades alternativas de Indígenas en Latinoamérica y principalmente en los países andinos, que tienen como fundamento y eje vertebrador a la Interculturalidad.

Lo que se pretende es que los estudiantes de la carrera de economía en la materia de proyecto I de la U.A.T.F: que tengan una interacción social universitaria mediante la cual ellos sean los artífices de la adquisición de conocimientos que les sirvan en el trascurrir de su vida profesional, ya que la propuesta promoverá a esta transformación; teniendo en cuenta que la extensión universitaria es todo el conjunto de la universidad y la interacción universidad sociedad más específico para un fin y la interacción social universitaria ya como el desarrollo de los estudiantes con los problemas de la sociedad y que se empañen de esta y sepan cómo poder resolverlos.

\section{Materia de Proyectos}

La materia de Proyectos I (ECO 411) del séptimo semestre, tiene las suficientes bases para desarrollar habilidades en los estudiantes para describir y ejercitar la metodología de formulación de proyectos, pero le falta el componente de interacción social ya que no cumple este componente, solo lo deja 
en simples ejercicios y lo lleva al entrono real y a las necesidades que tiene la población.

\section{CONCLUSIONES}

L a valoración de la institución universitaria conduce a concluir que se trata de una organización social e histórica que ha evolucionado desde un claustro encerrado en sí mismo, a una instancia social vinculada cada vez más con procesos extramuros asociados a problemas del entorno local. Concibe el desarrollo personal como una construcción cultural, que se realiza a través de la interacción con otras personas de una determinada cultura mediante la realización de actividades sociales compartidas, o sea el Enfoque Histórico Cultural.

La relación universidad-sociedad ha venido reflejando la evolución de las universidades con respecto a cómo han venido articulando su actividad con las del entorno y el impacto que dicha relación ha tenido en el desarrollo económico, social y político de la sociedad. La disciplina constituye así una oportunidad de diálogo con el entorno. No obstante, actualmente, dicho diálogo no se presenta de manera expedita, requiriéndose una concepción transformadora que propicie estrategias de cambio para promover la articulación de la disciplina con los problemas del desarrollo local.

Por su parte, la caracterización de la formación profesional de la carrera de economía, inmerso en su curricular la interacción y extensión universitaria solo apunta a dejar como mero recuerdo para la historia, la connotación de profesionales empíricos, para incursionar en la prospectiva moderna de un profesional actualizado, vinculado a los grandes retos del ser humano, la sociedad y el mundo, lo cual involucra, obviamente, la preocupación por el desarrollo profesional. Se tendrá que basar en la teoría de Vigotsky, principalmente en el aprendizaje sociocultural de cada individuo y por lo tanto en el medio en el cual se desarrolla para poder tener este profesional un carácter más cognoscitivo con el medio.

De esta manera se puede concluir que mediante la Interacción Social Universitaria el estudiante de Economía puede 
desarrollarse y tener una mejor precepción del perfil del profesional que se quiere llegar.

\section{REFERENCIAS}

Álvarez de Zayas, CM. (1996). La Universidad como Institución Social. Sucre, Bolivia: Universidad San Francisco Xavier

Azurduy, E. (1994). Reforma universitaria. Sucre, Bolivia: Editorial Universitaria

Centenario de la Universidad Autónoma Tomás Frías 1892-1992. (1993) Primera edición, Editores Extensión Universitaria, Impreso en editorial universitaria, Potosí Julio 1993

Chupajin, I. Y. (1977). Lógica formal. Ed: Universidad de Leningrado. Leningrado (en ruso)

Comité Ejecutivo de la Universidad Boliviana (1985). Documentos del VI Congreso Nacional de Universidades. Tarija, Bolivia: Editorial Universitaria

Comité Ejecutivo de la Universidad Boliviana (1998). IX Congreso Nacional de Universidades. Trinidad, Bolivia: Editorial Universitaria

Comité Ejecutivo de la Universidad Boliviana (1983). VI Congreso de Universidades. La Paz, Bolivia: Editorial Universitaria

Comité Ejecutivo de la Universidad Boliviana (1999). VIII Congreso de Universidades. La Paz, Bolivia: Editorial Universitaria

Curso Taller Metodología de la Investigación Educacional (2013), Universidad de Ciencias Pedagógicas Enrique José Varona, Dr. C. Calixto del Canto Colls

Federación Universitaria Boliviana (1929). Reglamento de debates. Estatuto Orgánico y Programa de la Federación Universitaria Boliviana. 2da. Ed. La Paz, Bolivia: Imprenta ATENEA;

Federación Universitaria de Docentes Universitarios de San Francisco Xavier. VII Congreso Nacional de Docentes Universitarios. Sucre, Bolivia: Federación de Docentes Universitarios de San Francisco Xavier 1998

La Nueva Ley Fundamental de la Universidad Boliviana. Ley pub. No 12972. (Nov 16, 1975)

Ley De La Educación Avelino Siñani - Elizardo Pérez Ley 070, 20 de Diciembre de 2010

Mao T.T (1980) Cinco tesis filosóficas. Lima, Perú: Lima S.A.

Medina EF. De la Universidad Popular a la Facultad Técnica. Sucre, Bolivia: Editorial Universitaria; 1999 
Secretaria Académica del Comité Ejecutivo de la Universidad Boliviana (1983). Bases epistemologías, teóricas, metodológicas y psicológicas del nuevo modelo académico. La Paz, Bolivia: Editorial Universitaria

Tavares L.A. Administración moderna de mantenimiento. Sucre, Bolivia: Editorial Universitaria; 1999

Universidad Autónomas Tomás Frías, Plan curricular de la carrera de economía, Documento del Sector Docente, Potosí Octubre de 2002

Universidad Boliviana XI Congreso nacional de universidades, La Paz, Bolivia Enero 2011 